\title{
Pulmonary Function and Arterial Stiffness in Chronic Heart Failure
}

\author{
Li Li, ${ }^{1}$ Bangchuan Hu, ${ }^{2}$ Shijin Gong, ${ }^{1}$ Yihua Yu, ${ }^{1}$ Haiwen Dai, ${ }^{1}$ and Jing Yan ${ }^{1}$ \\ ${ }^{1}$ Intensive Care Unit and Zhejiang Provincial Key Laboratory of Geriatrics, Zhejiang Hospital, 12 Lingyin Road, \\ Hangzhou 310013, China \\ ${ }^{2}$ Intensive Care Unit, Zhejiang Provincial People’s Hospital, 158 Shangtang Road, Hangzhou 310014, China
}

Correspondence should be addressed to Li Li; lilihbch@163.com and Jing Yan; yanjing2013@163.com

Received 30 September 2016; Revised 28 November 2016; Accepted 30 November 2016

Academic Editor: Fabrizio Montecucco

Copyright (C) $2016 \mathrm{Li} \mathrm{Li} \mathrm{et} \mathrm{al.} \mathrm{This} \mathrm{is} \mathrm{an} \mathrm{open} \mathrm{access} \mathrm{article} \mathrm{distributed} \mathrm{under} \mathrm{the} \mathrm{Creative} \mathrm{Commons} \mathrm{Attribution} \mathrm{License,} \mathrm{which}$ permits unrestricted use, distribution, and reproduction in any medium, provided the original work is properly cited.

\begin{abstract}
Arterial stiffness contributes to heart failure and is decreased by angiotensin receptor blockers (ARBs). This cross-sectional study aimed to assess associations of lung function and ARB with arterial stiffness in patients with chronic heart failure. 354 outpatients (168 males; 186 females; $68.2 \pm 7.2$ years old) with chronic heart failure were evaluated. Lung function parameters, including forced vital capacity (FVC), forced expiratory volume in $1 \mathrm{~s}\left(\mathrm{FEV}_{1}\right)$, and $\mathrm{FEV}_{1}$ to $\mathrm{FVC}$ ratio $\left(\mathrm{FEV}_{1} / \mathrm{FVC}\right)$, were assessed. The cardio-ankle vascular index (CAVI) was used to estimate arterial stiffness. Unadjusted correlation analyses revealed a positive association of CAVI with ARB but not ACEI, and a negative correlation with $\mathrm{FEV}_{1}(r=-0.2987, p<0.0001)$. Multiple stepwise regression analyses showed that ARB and FEV1 $(p<0.0001)$ were independent predicting factors for CAVI. These findings suggest that reduced pulmonary function is associated with increased CAVI. Pulmonary function protection could be used to improve the prognosis in heart failure, but additional studies are necessary.
\end{abstract}

\section{Introduction}

Increasing evidence demonstrates the positive associations of arterial stiffness with multiple cardiovascular diseases, including heart failure [1-3]. Meanwhile, arteriosclerosis progression increases arterial stiffness [4]. This in turn puts important pressure on the weakened heart, increasing the forward pressure wave signal, which precipitates ventricular ejection termination $[1,5]$. In addition, arterial stiffness affects diastolic function as well as ventricular relaxation [6]. Based on these findings, arterial stiffness currently constitutes a hot topic in the field of heart failure prevention research.

Impaired pulmonary function is well known in individuals suffering from chronic heart failure. For instance, a study assessing 20 stable ambulatory individuals presenting congestive heart failure caused by cardiomyopathy indicated that tidal expiratory flow limitation is common [7]. In addition, patients with stable mitral valve disease display reduced forced expiratory volume in $1 \mathrm{~s}\left(\mathrm{FEV}_{1}\right)$ and forced vital capacity (FVC), and increased residual volume (RV), which were associated with valvular disease severity [8]. The cardiothoracic ratio was shown to be inversely correlated with total lung capacity as well as with vital capacity [9]. Overall, patients with chronic heart failure present restrictive ventilatory defect and altered diffusing capacity. Previous reports suggested that altered pulmonary function is related to arterial stiffness in both healthy subjects and other population groups, including children [10] and patients with hypertension [11], diabetes, kidney ailments, and lung diseases [12]. However, the relationship between lung function and arterial stiffness in individuals suffering from chronic heart failure is currently unknown.

Multiple studies reported the protective effects of angiotensin receptor blockers (ARBs) against arterial stiffness [13]. Indeed, ARB treatment, as low-dose monotherapy or combined with a calcium channel blocker (CCB), has beneficial effects on arterial stiffness in patients with hypertension who do not achieve blood pressure control with routine monotherapy. However, the relation between ARB-reduced arterial stiffness and heart failure remains unclear.

Arterial stiffness is readily assessed by novel devices in clinic. Previous studies used pulse wave velocity as an index of arterial stiffness, but this index is influenced by blood pressure at the time of the measurement and may be 
influenced if blood pressure varies during the measurement [14]. A new index, namely, the cardio-ankle vascular index (CAVI), was developed for assessing arterial wall stiffness parameter [15]. As CAVI is independent of blood pressure, it is increasingly employed in evaluating arterial stiffness. The importance of arterial stiffness measured by CAVI in diabetes [16], atherosclerosis [17], hypertension [18], and kidney diseases [19] was revealed in several clinical studies, indicating that CAVI is high in atherosclerotic disease. Elevated PWV [1, 2, 20-22] and CAVI [23-25] were observed in people suffering from heart failure. However, the factors contributing to elevated CAVI in heart failure remain unclear.

Therefore, the current study aimed to assess potential associations among pulmonary function, ARB, and arterial stiffness as evaluated using CAVI instead of PWV in individuals suffering from chronic heart failure. We found that decreased pulmonary function is associated with elevated CAVI, while ARB was correlated to declined CAVI. Overall, pulmonary function protection and $A R B$ use may improve and ameliorate heart failure prognosis.

\section{Methods}

2.1. Ethical Approval. This study was approved by the ethics committee of Zhejiang Hospital, with exemption from informed consent requirement. It was according to the provisions of the Declaration of Helsinki (as revised in Brazil in 2013).

2.2. Study Population. From April 2008 to March 2010, 354 consecutive outpatients above 18 years, previously diagnosed with stable chronic heart failure, were recruited at the Heart Failure Clinic of Zhejiang Hospital. Chronic heart failure was diagnosed according to ESC and AHA/ACC recommendations [26]. Individuals with history of acute myocardial infarction or ischemic stroke in the past 3 months, dementia, severe chronic pulmonary disease, and end-stage kidney disease were excluded. In addition, patients with lung cancer and emphysema diagnosed by computerized tomography were also excluded.

2.3. Clinical and Biological Indexes. A standard questionnaire recording age, sex, smoking, drinking, comorbidities, medication, and medical history was used. The same experienced doctor assessed brachial blood pressure for all patients, three times consecutively by mercury sphygmomanometry, after at least $5 \mathrm{~min}$ of rest. Venous blood specimens were used for plasma glucose concentration measurements as well as serum level assessment of total cholesterol and triglycerides. Serum creatinine and plasma BNP and hs-CRP were assessed using routine techniques.

2.4. Echocardiography. M-mode and two-dimensional echocardiography were performed using an ultrasound system (Hewlett Packard, USA) equipped with a $2.5 \mathrm{MHz}$ transducer. M-mode echocardiography was used to assess left ventricular (LV) end-diastolic dimension (LVEDD) and LV endsystolic dimension (LVESD). LV ejection fraction (LVEF) was employed as an index of LV systolic function. LV diastolic function (LVDF) indices were next assessed by determining transmitral flow velocity using the routine Doppler echocardiographic technique; E velocity and A velocity were obtained as previously described [27], and E/A ratios were derived.

2.5. CAVI Measurement. CAVI was assessed on a VaSera VS1000 vascular screening system (Fukuda Denshi, Japan), as described previously [11]. This index helps assess stiffness of the aorta, femoral artery, and tibial artery and is in principle independent from blood pressure. After automatic measurement, right and left CAVI were obtained and averaged.

2.6. Lung Function Measurement. Lung function assays were carried out using a computerized spirometer (Yaeger, MSPET, Germany). The patient was in the sitting position, with nose clips. FVC and $\mathrm{FEV}_{1}$ from most accurate assays [28] were recorded. $\mathrm{FEV}_{1}$ to $\mathrm{FVC}$ ratios $\left(\mathrm{FEV}_{1} / \mathrm{FVC}\right)$ were calculated.

2.7. Statistical Analysis. The SAS software (SAS, USA) was used for all statistical analyses. Means and proportions were assessed by standard normal $z$-test and Fisher's exact test, respectively. Nonnormally distributed continuous data were submitted to logarithmic transformation for normalization. Variables significant in correlation analyses at $p<0.05$ were entered in multivariable linear analyses, performed to determine independent predictors of CAVI. A $p$ value below 0.05 was deemed to reflect statistical significance.

\section{Results}

3.1. Patient Features. Among the 363 individuals with diastolic heart failure, nine were excluded, including three who declined pulmonary function tests and six without satisfactory carotid waveforms. Thus, 354 cases were included in the final analyses. Their clinical features are reported by gender in Table 1 . The 168 male and 186 female patients had similar ages; no significant differences were observed in systolic and diastolic blood pressure, total cholesterol level, E/A ratio, hypertension, and atrial fibrillation diagnoses, as well as the percentages of patients taking ACEI/ARB, beta-blockers, diuretics, and aspirin (all $p>0.05$ ). Compared with women, men had higher BMI, hs-CRP, serum triglycerides, and creatinine and LVEDD, LVESD, FVC, FEV 1 , and coronary heart disease rates, with lower heart rate, BNP, fasting glucose, prevalence of diabetes mellitus, $\mathrm{FEV}_{1}$, and $\mathrm{FEV}_{1} / \mathrm{FVC}$, as well as CAVI (all $p<0.05$ ). Of the 168 men, 33 (19.6\%) were current smokers ( $>1$ cigarette/day, $>1$ year) and $51(30.4 \%)$ had consumed alcohol for $>10$ years (alcohol intake $>5 \mathrm{~g} /$ day). Less women had smoking $(12,6.4 \%)$ and drinking $(6,3.2 \%)$ habits (both $p<0.05$ ).

\subsection{Association of Arterial Stiffness Parameters with Clinical} Indexes. The associations of CAVI parameters with other clinical indexes in all subjects are summarized in Tables 2 and 3. Univariate analyses demonstrated positive associations of CAVI with age $(r=0.346)$, systolic blood pressure $(r=$ 
TABLE 1: Clinical characteristics of the participants in chronic heart failure.

\begin{tabular}{|c|c|c|c|c|}
\hline Clinical characteristic & All patients $(n=354)$ & Male $(n=168)$ & Female $(n=186)$ & $p$ value \\
\hline Age (years) & $68.2 \pm 7.2$ & $69.1 \pm 6.9$ & $67.4 \pm 7.4$ & 0.2887 \\
\hline Body mass index $\left(\mathrm{kg} / \mathrm{m}^{2}\right)$ & $25.99 \pm 3.20$ & $26.03 \pm 2.40$ & $25.95 \pm 3.80$ & $<0.0001$ \\
\hline Systolic BP (mmHg) & $130.7 \pm 7.0$ & $130.1 \pm 7.5$ & $131.5 \pm 6.4$ & 0.0601 \\
\hline Diastolic BP (mmHg) & $79.2 \pm 7.3$ & $80.2 \pm 6.8$ & $78.4 \pm 7.8$ & 0.1040 \\
\hline Heart rate (beats/min) & $73.5 \pm 9.7$ & $72.8 \pm 8.6$ & $74.2 \pm 10.5$ & 0.0118 \\
\hline Current smoking, $n(\%)$ & $39(11.0 \%)$ & $33(19.6 \%)$ & $6(3.2 \%)$ & $<0.0001$ \\
\hline Alcohol intake, $n(\%)$ & $63(17.8 \%)$ & $51(30.4 \%)$ & $12(6.4 \%)$ & $<0.0001$ \\
\hline $\mathrm{BNP}(\mathrm{pg} / \mathrm{mL})$ & 138 (96 to 152$)$ & 131 (114 to 151$)$ & 149 (88 to 154$)$ & $<0.0001$ \\
\hline hs-CRP (mmol/L) & $2.08 \pm 2.41$ & $2.16 \pm 2.21$ & $1.99 \pm 2.61$ & 0.0296 \\
\hline \multicolumn{5}{|l|}{ Biochemical parameters } \\
\hline Fasting glucose (mmol/L) & $5.81 \pm 1.49$ & $5.62 \pm 1.09$ & $5.99 \pm 1.62$ & $<0.0001$ \\
\hline Triglyceride $(\mathrm{mmol} / \mathrm{L})$ & $1.89 \pm 1.10$ & $1.93 \pm 1.28$ & $1.84 \pm 0.90$ & $<0.0001$ \\
\hline Total cholesterol (mmol/L) & $5.21 \pm 0.99$ & $4.99 \pm 0.92$ & $5.41 \pm 1.00$ & 0.2530 \\
\hline $\mathrm{HDL}(\mathrm{mmol} / \mathrm{L})$ & $1.01 \pm 0.09$ & $0.96 \pm 0.08$ & $1.00 \pm 0.10$ & 0.3650 \\
\hline $\mathrm{LDL}(\mathrm{mmol} / \mathrm{L})$ & $3.28 \pm 0.58$ & $3.44 \pm 0.56$ & $3.24 \pm 0.60$ & 0.4750 \\
\hline VLDL (mmol/L) & $0.26 \pm 0.19$ & $0.30 \pm 0.22$ & $0.24 \pm 0.17$ & 0.6010 \\
\hline Serum creatinine $(\mu \mathrm{mol} / \mathrm{L})$ & $74.3 \pm 18.3$ & $87.4 \pm 15.9$ & $62.4 \pm 10.5$ & $<0.0001$ \\
\hline \multicolumn{5}{|l|}{ Related disorders } \\
\hline Hypertension, $n(\%)$ & $255(72.0)$ & $118(70.4)$ & $137(74.0)$ & 0.4753 \\
\hline Diabetes mellitus, $n(\%)$ & $81(22.0)$ & $27(16.1)$ & $54(29.0)$ & 0.0037 \\
\hline Atrial fibrillation, $n(\%)$ & $30(8.5)$ & $12(7.2)$ & $18(9.7)$ & 0.4086 \\
\hline Coronary heart disease, $n(\%)$ & $132(37.3)$ & $78(46.4)$ & $54(29.0)$ & 0.0359 \\
\hline \multicolumn{5}{|l|}{ Treatment drugs } \\
\hline ACEI/ARB, $n(\%)$ & $180(50.8)$ & $96(57.2)$ & $84(45.2)$ & 0.3749 \\
\hline Beta-blockers, $n(\%)$ & $135(38.1)$ & $63(37.5)$ & $72(38.7)$ & 0.9095 \\
\hline Diuretics, $n(\%)$ & $165(46.6)$ & $105(62.5)$ & $60(32.5)$ & 0.0506 \\
\hline Aspirin, $n(\%)$ & $138(39.0)$ & $60(35.6)$ & $78(42.0)$ & 0.4607 \\
\hline Statin, $n(\%)$ & $54(15.3)$ & $42(25.0)$ & $12(6.4)$ & 0.0010 \\
\hline \multicolumn{5}{|l|}{ Echocardiography parameters } \\
\hline $\mathrm{LAD}(\mathrm{mm})$ & $32.9 \pm 4.0$ & $33.6 \pm 4.1$ & $32.2 \pm 3.9$ & 0.4933 \\
\hline $\operatorname{LVEDD}(\mathrm{mm})$ & $48.1 \pm 4.9$ & $49.7 \pm 5.2$ & $47.0 \pm 4.0$ & 0.0005 \\
\hline $\operatorname{LVESD}(\mathrm{mm})$ & $30.7 \pm 4.9$ & $31.9 \pm 5.3$ & $29.6 \pm 3.5$ & $<0.0001$ \\
\hline Ejection fraction, $\%$ & $55.3 \pm 7.5$ & $55.3 \pm 8.2$ & $55.9 \pm 6.9$ & 0.0169 \\
\hline $\mathrm{E} / \mathrm{A}$ ratio & $0.74 \pm 0.18$ & $0.73 \pm 0.17$ & $0.75 \pm 0.18$ & 0.3784 \\
\hline \multicolumn{5}{|l|}{ Pulmonary function } \\
\hline FVC (L) & $2.76 \pm 0.82$ & $3.06 \pm 0.81$ & $2.28 \pm 0.56$ & $<0.0001$ \\
\hline FEV1 (L) & $2.16 \pm 1.50$ & $2.44 \pm 1.88$ & $1.76 \pm 0.54$ & $<0.0001$ \\
\hline FEV1/FVC ratio & $75.66 \pm 11.48$ & $75.24 \pm 10.21$ & $76.29 \pm 13.10$ & 0.0211 \\
\hline CAVI & $8.57 \pm 1.10$ & $8.47 \pm 1.10$ & $8.66 \pm 1.09$ & 0.0005 \\
\hline
\end{tabular}

Values are mean \pm SD. $p$ values reflect the differences between males and females.

ACEI: angiotensin converting enzyme inhibitor; ARB: angiotensin receptor blocker; LAD: left atrial diameter; LVEDD: left ventricular end-diastolic diameter; LVESD: left ventricular end-systolic diameter; E/A ratio: ratio of early transmitral flow velocity to atrial flow velocity; BNP: B-type natriuretic peptide; hs-CRP: high-sensitivity C-reactive protein; FVC: forced volume capacity; FEV1: forced expiratory volume in 1s; CAVI: cardio-ankle vascular index.

$0.119)$, hs-CRP $(r=0.216)$, and BNP $(r=0.212)$ (all $p<$ $0.05)$, and negative associations with body mass index (BMI, $r=-0.204)$, FVC $(r=-0.271)$, E/A ratio $(r=-0.176)$, and FEV1 $(r=-0.2986)($ all $p<0.001$; Table 2$)$.

Multiple stepwise regression was carried out for the identification of clinical indexes independently predicting CAVI. Interestingly, age $(\beta=0.039 \pm 0.009), \operatorname{BMI}(\beta=-0.057$ $\pm 0.0174), \operatorname{ARB}(\beta=-0.301 \pm 0.129), \operatorname{FEV} 1(\beta=-0.525 \pm 0.112)$, $\mathrm{E} / \mathrm{A}$ ratio $(\beta=-0.808 \pm 0.337)$, and $\mathrm{BNP}(\beta=-0.006 \pm 0.002)$ (all $p<0.05$ ) were found to be independent determinants of CAVI (Table 3). After adjustment for medication and history of hypertension and coronary heart disease, similar results were obtained (data not shown).

\section{Discussion}

In this report, we showed that pulmonary function and ARB were significantly associated with changes of arterial stiffness 
TABLE 2: Correlation analyses for CAVI.

\begin{tabular}{lcc}
\hline & $R$ & $p$ \\
\hline Age & 0.346 & $<0.001$ \\
Gender & 0.086 & 0.104 \\
Body mass index & -0.204 & $<0.001$ \\
Systolic BP & 0.119 & 0.026 \\
Diastolic BP & -0.085 & 0.114 \\
Current smoking & 0.065 & 0.220 \\
hs-CRP & 0.216 & $<0.001$ \\
Hypertension & -0.014 & 0.790 \\
Diabetes mellitus & 0.050 & 0.320 \\
Coronary heart disease & 0.008 & 0.878 \\
ACEI & 0.028 & 0.600 \\
ARB & -0.100 & 0.056 \\
FVC & -0.271 & 0.030 \\
FEV1 & -0.299 & $<0.001$ \\
FEV1/FVC ratio & -0.048 & 0.376 \\
LVEF & -0.006 & 0.917 \\
E/A ratio & -0.176 & 0.001 \\
BNP & 0.212 & $<0.001$ \\
\hline ACEI angiotensio converting
\end{tabular}

ACEI: angiotensin converting enzyme inhibitor; ARB: angiotensin receptor blocker; LAD: left atrial diameter; LVEDD: left ventricular end-diastolic diameter; LVESD: left ventricular end-systolic diameter; E/A ratio: ratio of early transmitral flow velocity to atrial flow velocity; BNP: B-type natriuretic peptide; hs-CRP: high-sensitivity C-reactive protein; FVC: forced volume capacity; FEV1: forced expiratory volume in $1 \mathrm{~s}$; CAVI: cardio-ankle vascular index.

TABLE 3: Multivariate linear analyses for CAVI.

\begin{tabular}{lcc}
\hline & $\beta \pm \mathrm{SE}$ & $p$ \\
\hline Age & $0.039 \pm 0.009$ & $<0.001$ \\
Body mass index & $-0.057 \pm 0.017$ & $<0.001$ \\
ARB & $-0.301 \pm 0.129$ & 0.020 \\
FEV1 & $-0.525 \pm 0.112$ & $<0.001$ \\
E/A ratio & $-0.808 \pm 0.337$ & 0.017 \\
BNP & $-0.006 \pm 0.002$ & 0.0046 \\
\hline
\end{tabular}

ARB: angiotensin receptor blocker; $\mathrm{E} / \mathrm{A}$ ratio: ratio of early transmitral flow velocity to atrial flow velocity; BNP: B-type natriuretic peptide; hFEV1: forced expiratory volume in $1 \mathrm{~s}$.

monitored with CAVI in subjects suffering from chronic heart failure, after adjustment for potential confounders.

Pulse wave velocity is an index of arterial stiffness, but this index is influenced by blood pressure at the time of the measurement and may be influenced if blood pressure varies during the measurement [14]. On the other hand, CAVI is independent of blood pressure and its importance in relation to arterial stiffness has been shown in diabetes [16], atherosclerosis [17], hypertension [18], and kidney diseases [19]. Elevated PWV [1, 2, 20-22] and CAVI [23-25] were observed in people suffering from heart failure.

A number of factors are known to affect lung function, such as training, age, height, asthma, and sex, among others $[29,30]$. All of these factors can affect the final result of $\mathrm{FEV}_{1}$. The relationship between lung function and arterial stiffness has been widely studied over the past few years. A previous report found that $\mathrm{FEV}_{1}$ was negatively correlated with arterial stiffness, which was still significant after adjusting for age, height, and cardiovascular risk factors [31]. In addition, recent findings revealed an inverse correlation of $\mathrm{FEV}_{1}$ and carotidfemoral pulse wave velocity (CFPWV) in elderly patients [32]. Interestingly, it was found that individuals suffering from COPD showed significantly higher CFPWV values than control subjects [33]. Meanwhile, a trial assessing individuals above 20 years of age indicated a significant association of decreased $\mathrm{FEV}_{1}$ with enhanced arterial stiffness measured by peripheral pulse pressure in the general population [34]. In a report assessing 45 hypertensive outpatients, another measure of arterial stiffness, CAVI, was independently associated with $\mathrm{FEV}_{1} / \mathrm{FVC}$ [11]. However, others found discrepant data. The Seattle Nikkei Health Study did not observe a correlation between abnormal lung function and aortic PWV [35]. Different clinical characteristics of participants may account for the discrepancies among studies. Our study firstly showed CAVI was significantly associated with $\mathrm{FEV}_{1}$ in individuals suffering from chronic heart failure after adjusting for confounding factors, confirming an evident association of pulmonary function with arterial stiffness. Nevertheless, as stated above, many factors can affect $\mathrm{FEV}_{1}$ and additional studies with a larger sample size are necessary to rule out the possible interference of these factors on the association between arterial stiffness and $\mathrm{FEV}_{1}$.

The mechanisms explaining the associations between pulmonary function and arterial stiffness in subjects suffering from chronic heart failure remain largely unknown. Excessive inflammatory responses contribute to arterial injury, which could cause vascular changes and stiffness [36]. In addition, oxidative stress and endothelium dysfunction pathogenetically contribute to the negative correlation between lung function and arterial stiffness in chronic heart failure [37,38]. One may exert an influence on the other or they could be all affected by external factors, such as patient habits and metabolic disruption. This intrinsic association of pulmonary function with arterial stiffness in individuals suffering from heart failure merits further study.

Multiple reports confirmed the protective impact of ARB on arterial stiffness in essential hypertension and diabetes $[39,40]$. Guidelines have been provided for heart failure treatment with ACE inhibitors or ARB [41]. However, conflicting data were obtained in randomized controlled trials comparing ARB and ACEI. While the ELITE I study indicated better outcomes of ARB treatment, the ELITE II study indicated similar efficacy for heart failure [42]. This study indicated a relationship between ARB and arterial stiffness, which suggested a potential advantage of ARB in chronic heart failure patients, especially those with arterial stiffness. $\mathrm{ARB}$ as an alternative of ACEI may need reconsideration, but additional studies are necessary to address specifically this issue.

A few limitations of this study should be mentioned. First and foremost, it had a cross-sectional design, which cannot determine the chronological order or cause-to-effect relationship among pulmonary function decline, ARB use, and arterial stiffness. Therefore, prospective studies are needed. 
In addition, we did not distinguish individuals with preserved or reduced LVEF among subjects suffering from heart failure. Furthermore, all enrolled subjects could perform the pulmonary function test; thus, these findings might not reflect the situation of patients with severe heart failure. There remained the possibility that there might be some different backgrounds between patients with ARB and those with ACEI. Lastly, the sample size was relatively small, and the current data need to be confirmed in larger populations.

\section{Conclusion}

Pulmonary function and ARB could be associated with arterial stiffness monitored with CAVI in individuals suffering from chronic heart failure. Pulmonary function protection could be used to improve the prognosis in heart failure, but additional studies are necessary.

\section{Competing Interests}

The authors declare that there are no competing interests regarding the publication of this paper.

\section{Acknowledgments}

This work was funded by the National Natural Science Foundation of China (no. 81401580), Zhejiang Province Natural Science Foundation (no. LY14H150006), and the Health and Family Planning Commission of Zhejiang Province (no. 2013KYA002).

\section{References}

[1] H. Ooi, W. Chung, and A. Biolo, "Arterial stiffness and vascular load in heart failure," Congestive Heart Failure, vol. 14, no. 1, pp. 31-36, 2008.

[2] D. A. Duprez, "Arterial stiffness/elasticity in the contribution to progression of heart failure," Heart Failure Clinics, vol. 8, no. 1, pp. 135-141, 2012.

[3] P. Palatini, E. Casiglia, J. Gasowski et al., "Arterial stiffness, central hemodynamics, and cardiovascular risk in hypertension," Vascular Health and Risk Management, vol. 7, no. 1, pp. 725-739, 2011.

[4] N. M. Van Popele, D. E. Grobbee, M. L. Bots et al., "Association between arterial stiffness and atherosclerosis: The Rotterdam Study," Stroke, vol. 32, no. 2, pp. 454-460, 2001.

[5] C. W. Tsao, A. Lyass, M. G. Larson et al., "Relation of central arterial stiffness to incident heart failure in the community," Journal of the American Heart Association, vol. 4, no. 11, Article ID e002189, 2015.

[6] L. Agoşton-Coldea, T. Mocan, and C. Bobar, "Arterial stiffness and left ventricular diastolic function in the patients with hypertension," Romanian Journal of Internal Medicine, vol. 46, no. 4, pp. 313-321, 2008.

[7] R. Torchio, C. Gulotta, P. Greco-Lucchina et al., "Orthopnea and tidal expiratory flow limitation in chronic heart failures," Chest, vol. 130, no. 2, pp. 472-479, 2006.

[8] G. C. Dogliotti, P. F. Angelino, A. Brusca et al., "Pulmonary function in mitral valve disease. Hemodynamic and ventilatory studies," The American Journal of Cardiology, vol. 3, no. 1, pp. 28-39, 1959.

[9] J. K. Shim, D. H. Chun, Y. S. Choi, J. Y. Lee, S. W. Hong, and Y. L. Kwak, "Effects of early vital capacity maneuver on respiratory variables during multivessel off-pump coronary artery bypass graft surgery," Critical Care Medicine, vol. 37, no. 2, pp. 539-544, 2009.

[10] J. G. Ayer, E. G. Belousova, J. A. Harmer, B. Toelle, D. S. Celermajer, and G. B. Marks, "Lung function is associated with arterial stiffness in children," PLOS ONE, vol. 6, no. 10, Article ID e26303, 2011.

[11] H. Masugata, S. Senda, H. Okada et al., "Association between arterial stiffness and pulmonary function in hypertensive patients," Hypertension Research, vol. 35, no. 4, pp. 388-392, 2012.

[12] D. A. McAllister, J. D. Maclay, N. L. Mills et al., "Arterial stiffness is independently associated with emphysema severity in patients with chronic obstructive pulmonary disease," American Journal of Respiratory and Critical Care Medicine, vol. 176, no. 12, pp. 1208-1214, 2007.

[13] F. Peng, H. Pan, B. Wang, J. Lin, and W. Niu, "The impact of angiotensin receptor blockers on arterial stiffness: a metaanalysis," Hypertension Research, vol. 38, no. 9, pp. 613-620, 2015.

[14] T. Pereira, C. Correia, and J. Cardoso, "Novel methods for pulse wave velocity measurement," Journal of Medical and Biological Engineering, vol. 35, no. 5, pp. 555-565, 2015.

[15] K. Shirai, J. Utino, K. Otsuka, and M. Takata, "A novel blood pressure-independent arterial wall stiffness parameter; cardioankle vascular index (CAVI)," Journal of Atherosclerosis and Thrombosis, vol. 13, no. 2, pp. 101-107, 2006.

[16] H. Wang, J. Liu, H. Zhao et al., "Arterial stiffness evaluation by cardio-ankle vascular index in hypertension and diabetes mellitus subjects," Journal of the American Society of Hypertension, vol. 7, no. 6, pp. 426-431, 2013.

[17] K. Kadota, N. Takamura, K. Aoyagi et al., "Availability of cardio-ankle vascular index (CAVI) as a screening tool for atherosclerosis," Circulation Journal, vol. 72, no. 2, pp. 304-308, 2008.

[18] T. Okura, S. Watanabe, M. Kurata et al., "Relationship between cardio-ankle vascular index (CAVI) and carotid atherosclerosis in patients with essential hypertension," Hypertension Research, vol. 30, no. 4, pp. 335-340, 2007.

[19] T. Namikoshi, S. Fujimoto, D. Yorimitsu et al., "Relationship between vascular function indexes, renal arteriolosclerosis, and renal clinical outcomes in chronic kidney disease," Nephrology, vol. 20, no. 9, pp. 585-590, 2015.

[20] A. P. Patrianakos, F. I. Parthenakis, E. Nyktari, N. Malliaraki, D. N. Karakitsos, and P. E. Vardas, "Central aortic stiffness in patients with nonischemic dilated cardiomyopathy: relationship with neurohumoral activation," Journal of Cardiac Failure, vol. 15, no. 8, pp. 665-672, 2009.

[21] S. Shibata, J. L. Hastings, A. Prasad et al., "Congestive heart failure with preserved ejection fraction is associated with severely impaired dynamic Starling mechanism," Journal of Applied Physiology, vol. 110, no. 4, pp. 964-971, 2011.

[22] S. Kang, H.-M. Fan, J. Li et al., "Relationship of arterial stiffness and early mild diastolic heart failure in general middle and aged population," European Heart Journal, vol. 31, no. 22, pp. 27992807, 2010.

[23] C. Zhang, M. Ohira, T. Iizuka et al., "Cardio-ankle vascular index relates to left ventricular ejection fraction in patients with 
heart failure: a retrospective study," International Heart Journal, vol. 54, no. 4, pp. 216-221, 2013.

[24] C.-K. Sun, "Cardio-ankle vascular index (CAVI) as an indicator of arterial stiffness," Integrated Blood Pressure Control, vol. 6, pp. 27-38, 2013.

[25] N. Satoh-Asahara, K. Kotani, H. Yamakage et al., "Cardioankle vascular index predicts for the incidence of cardiovascular events in obese patients: a multicenter prospective cohort study (Japan Obesity and Metabolic Syndrome Study: JOMS)," Atherosclerosis, vol. 242, no. 2, pp. 461-468, 2015.

[26] C. W. Yancy, M. Jessup, B. Bozkurt et al., "2013 ACCF/AHA guideline for the management of heart failure: a report of the american college of cardiology foundation/american heart association task force on practice guidelines," Circulation, vol. 128, no. 16, pp. e240-e327, 2013.

[27] S. Noguchi, H. Masugata, S. Senda et al., "Correlation of arterial stiffness to left ventricular function in patients with reduced ejection fraction," Tohoku Journal of Experimental Medicine, vol. 225, no. 3, pp. 145-151, 2011.

[28] M. P. Swanney, G. Ruppel, P. L. Enright et al., "Using the lower limit of normal for the FEV1/FVC ratio reduces the misclassification of airway obstruction," Thorax, vol. 63, no. 12, pp. 1046-1051, 2008.

[29] S. Ostrowski and W. Barud, "Factors influencing lung function: are the predicted values for spirometry reliable enough?" Journal of Physiology and Pharmacology, vol. 57, no. 4, pp. 263271, 2006.

[30] P. S. Bakke, "Factors affecting growth of FEV1," Monaldi Archives for Chest Disease, vol. 59, no. 2, pp. 103-107, 2003.

[31] D. A. Duprez, M. O. Hearst, P. L. Lutsey et al., "Associations among lung function, arterial elasticity, and circulating endothelial and inflammation markers: the multiethnic study of atherosclerosis," Hypertension, vol. 61, no. 2, pp. 542-548, 2013.

[32] C. E. Bolton, J. R. Cockcroft, R. Sabit et al., "Lung function in mid-life compared with later life is a stronger predictor of arterial stiffness in men: The Caerphilly Prospective Study," International Journal of Epidemiology, vol. 38, no. 3, pp. 867876, 2009.

[33] R. Sabit, C. E. Bolton, P. H. Edwards et al., "Arterial stiffness and osteoporosis in chronic obstructive pulmonary disease," American Journal of Respiratory and Critical Care Medicine, vol. 175, no. 12, pp. 1259-1265, 2007.

[34] M. D. Jankowich, T. Taveira, and W.-C. Wu, "Decreased lung function is associated with increased arterial stiffness as measured by peripheral pulse pressure: data from NHANES III," American Journal of Hypertension, vol. 23, no. 6, pp. 614-619, 2010.

[35] K. Taneda, T. Namekata, D. Hughes, K. Suzuki, R. Knopp, and K. Ozasa, "Association of lung function with atherosclerotic risk factors among japanese americans: Seattle Nikkei Health Study," Clinical and Experimental Pharmacology and Physiology, vol. 31, S2, pp. S31-S34, 2004.

[36] K. G. Moulakakis, S. N. Mylonas, J. Kakisis et al., "Arterial stiffness alterations and inflammatory response following endovascular aortic repair: based on a presentation at the 2013 VEITH Symposium, November 19-23, 2013 (New York, NY, USA)," Aorta, vol. 3, no. 2, pp. 75-80, 2015.

[37] C. N. Marti, M. Gheorghiade, A. P. Kalogeropoulos, V. V. Georgiopoulou, A. A. Quyyumi, and J. Butler, "Endothelial dysfunction, arterial stiffness, and heart failure," Journal of the American College of Cardiology, vol. 60, no. 16, pp. 1455-1469, 2012.
[38] T. J. Anderson, "Arterial stiffness or endothelial dysfunction as a surrogate marker of vascular risk," Canadian Journal of Cardiology B, vol. 22, pp. 72B-80B, 2006.

[39] J. Agata, D. Nagahara, S. Kinoshita et al., "Angiotensin II receptor blocker prevents increased arterial stiffness in patients with essential hypertension," Circulation Journal, vol. 68, no. 12, pp. 1194-1198, 2004.

[40] J. Karalliedde, A. Smith, L. Deangelis et al., "Valsartan improves arterial stiffness in type 2 diabetes independently of blood pressure lowering," Hypertension, vol. 51, no. 6, pp. 1617-1623, 2008.

[41] C. W. Yancy, M. Jessup, B. Bozkurt et al., "2016 ACC/AHA/ HFSA focused update on new pharmacological therapy for heart failure: an update of the 2013 ACCF/AHA guideline for the management of heart failure: a report of the American College of Cardiology/American Heart Association Task Force on Clinical Practice Guidelines and the Heart Failure Society of America," Journal of the American College of Cardiology, vol. 68, no. 13, pp. 1476-1488, 2016.

[42] T. K. W. Ma, K. K. H. Kam, B. P. Yan, and Y.-Y. Lam, "Reninangiotensin-aldosterone system blockade for cardiovascular diseases: current status," British Journal of Pharmacology, vol. 160, no. 6, pp. 1273-1292, 2010. 


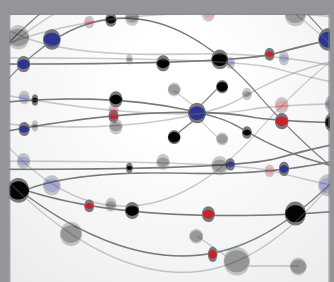

The Scientific World Journal
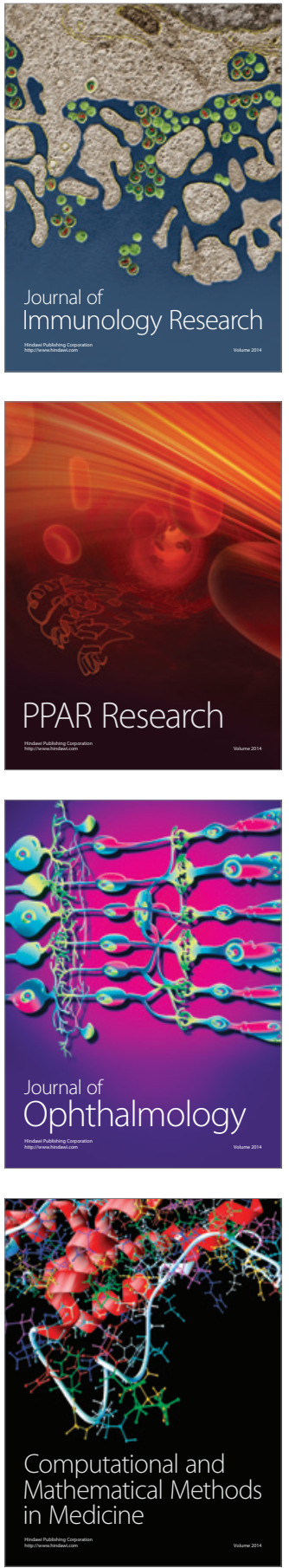

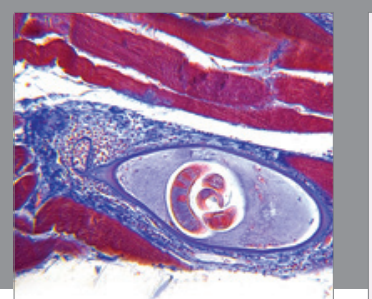

Gastroenterology Research and Practice

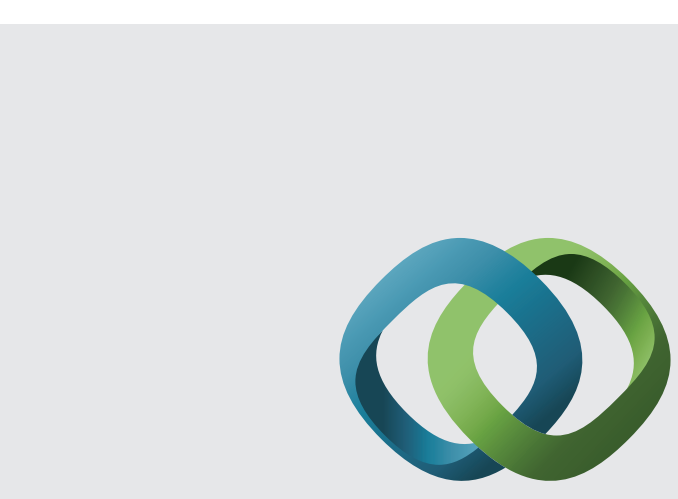

\section{Hindawi}

Submit your manuscripts at

http://www.hindawi.com
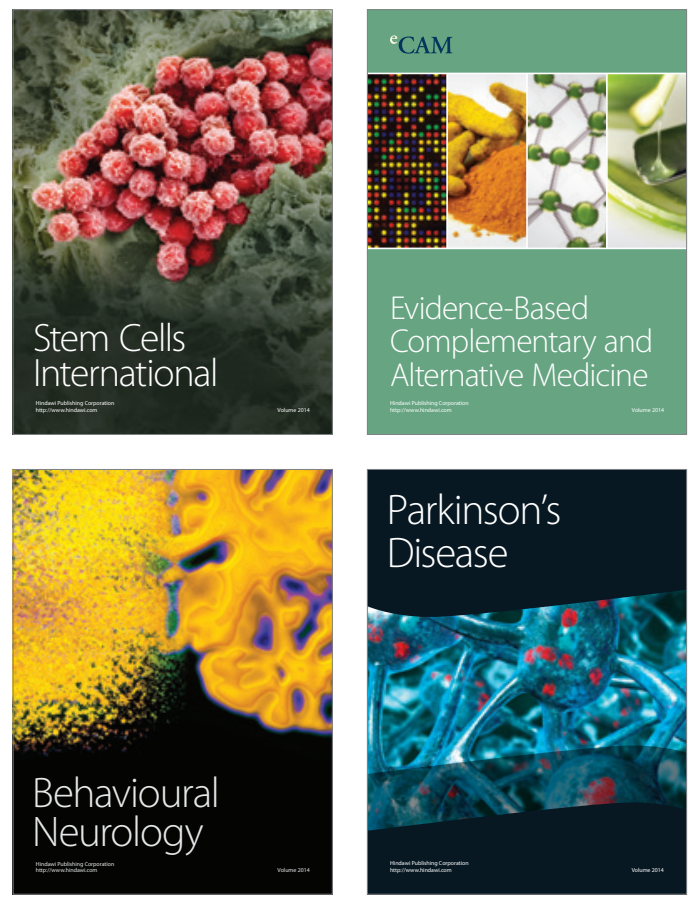
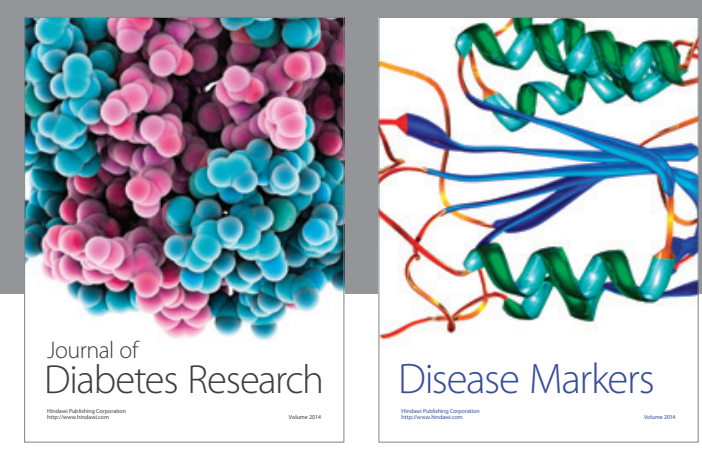

Disease Markers
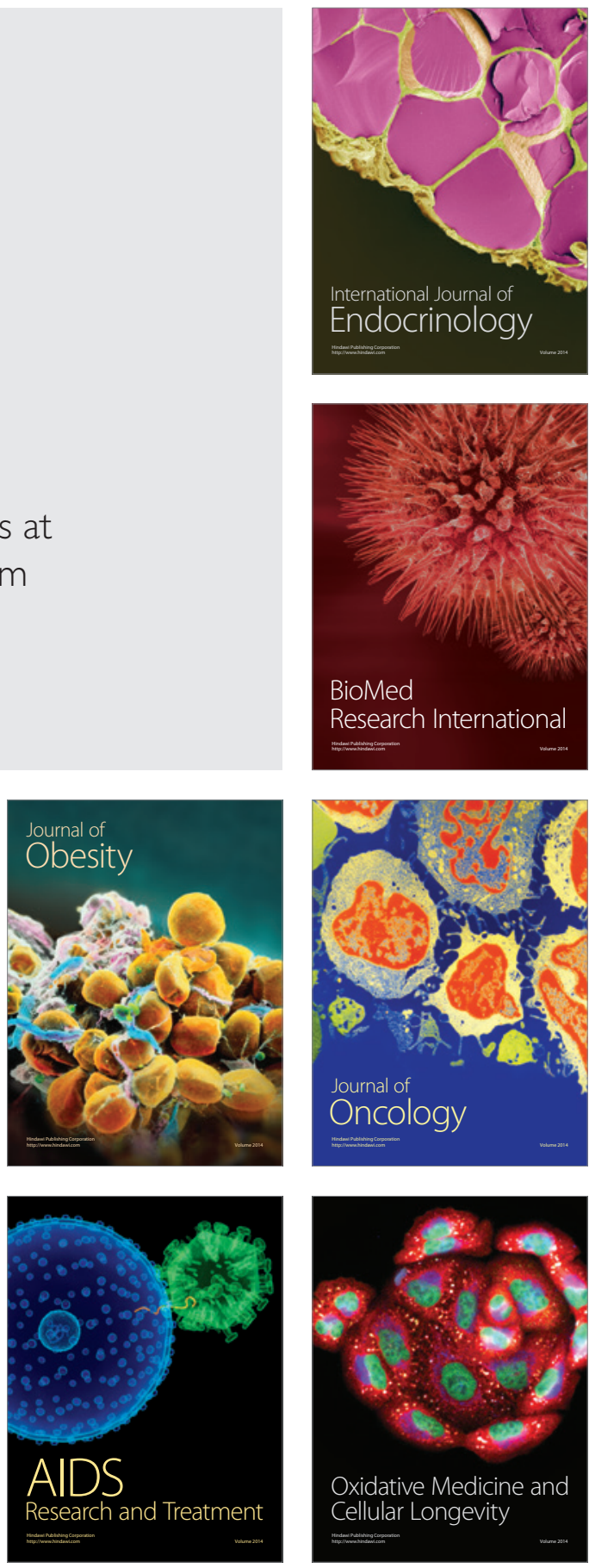